\title{
A Case Series on Suspected Remdesivir Induced Hyperammonemia
}

\author{
Sagar R. Bhimani, Sapna B. Gupta, Kamlesh P. Patel, and Supriya D. Malhotra
}

\section{ABSTRACT}

Remdesivir is a broad spectrum anti-viral drug that has shown to inhibit SARS-CoV-2. In absence of any effective treatment for SARS-CoV-2 infection (COVID-19), Remdesivir has been tried for a compassionate use in severe COVID-19. Remdesivir has shown promise in the management of patients with COVID-19 although recent studies have shown concerns with its effectiveness and safety in practice. Despite this there is a need to document potential Adverse drug Reaction (ADR) to guide future decisions. We describe 2 cases of Suspected Remdesivir-induced hyperammonemia (SRIH) in patients with normal liver function. Serum ammonia levels was raised much above the baseline after a fortnight of therapy. After completion of the therapy, there was gradual improvement and normalization of serum ammonia levels. This suggested a causal relationship which was possibly due to the administration of Remdesivir drug. With the increasing use, physicians should be aware of this potential ADR of Remdesivir and evaluate ammonia levels in patients taking Remdesivir who present with alterations in mental status.

Keywords: Adverse drug reaction, COVID-19, Hyperammonemia, Remdesivir, SARS-Co-V-2.

\section{INTRODUCTION}

Remdesivir is an adenosine analogue that has a broadspectrum antiviral activity against several viruses such as respiratory syncytial virus, Nipah virus, Ebola virus (EBOV), Middle East respiratory syndrome (MERS-CoV), and Severe Acute respiratory Syndrome Coronavirus-1 (SARS-CoV-1) [1]-[3] In current pandemic situation increasing use of Remdesivir for treating moderate to severe novel coronavirus disease 2019 (COVID-19).

The primary mechanism of Remdesivir is the incorporation of the nucleoside triphosphate GS-443902 into nascent RNA chains by viral RNA-dependent RNA polymerase, causing delayed RNA chain termination during the process of viral replication [4]. In summary, Remdesivir is a prodrug and inhibits viral RNA polymerases and decreases viral RNA production when intracellularly metabolized to an ATP analogue, this results in the termination of RNA transcription and decreases viral RNA production [4].

It is important to continue to collect safety data on the repurposed use of Remdesivir for the treatment of patients with COVID-19 alongside the collection of additional data regarding its effectiveness in patients with moderate to severe disease.

The common adverse events noted during compassionate use of Remdesivir in patients with COVID-19 are rash, diarrhea, hypotension, abnormal liver function, increase literature.

\section{A. Case 1} Pneumonitis.
Published Online: July 11, 2021

ISSN: $2736-5476$

DOI: $10.24018 /$ ejclinicmed.2021.2.3.96

\section{S. R. Bhimani}

Smt. NHL Municipal Medical College, Ahmedabad, Gujarat, India.

(e-mail: sagar.bhimani843@gmail.com) S. D. Gupta

Smt. NHL Municipal Medical College, Ahmedabad, Gujarat, India.

(e-mail: sapna_gupta76@yahoo.com)

K. P. Patel

Smt. NHL Municipal Medical College, Ahmedabad, Gujarat, India.

(e-mail: drkamleshp@yahoo.com)

S. D. Malhotra*

Smt. NHL Municipal Medical College, Ahmedabad, Gujarat, India.

(e-mail: supriyadmalhotra ${ }^{\circledR}$ gmail.com)

*Corresponding Author

serum aminotransferase and renal impairment [5]. Suspected Remdesivir-induced hyperammonemia (SRIH) is serious adverse drug reaction which is not well documented in

Here we present two case reports of SRIH both occurring in patients with no history of underlying liver disease and its normalization when treatment was completed.

These case reports of nonhepatic hyperammonemia, i.e., elevated serum ammonia secondary to a nonhepatic etiology have been rarely reported for Remdesivir. This information can further guide physicians and others, for its use as well as safety of patients in management of COVID-19.

\section{OBSERVATIONS}

An 80 years old hypertensive and hypothyroid male patient was brought to Emergency Medicine Department of tertiary care hospital with complains of cough for 4 days which was dry in nature, weakness for 2 days, low grade fever for 2 days and breathing difficulty for 1 day. No past history of Tuberculosis, COPD, Asthma, IHD, CKD and no surgical past history. Patient was diagnosed with COVID-19 


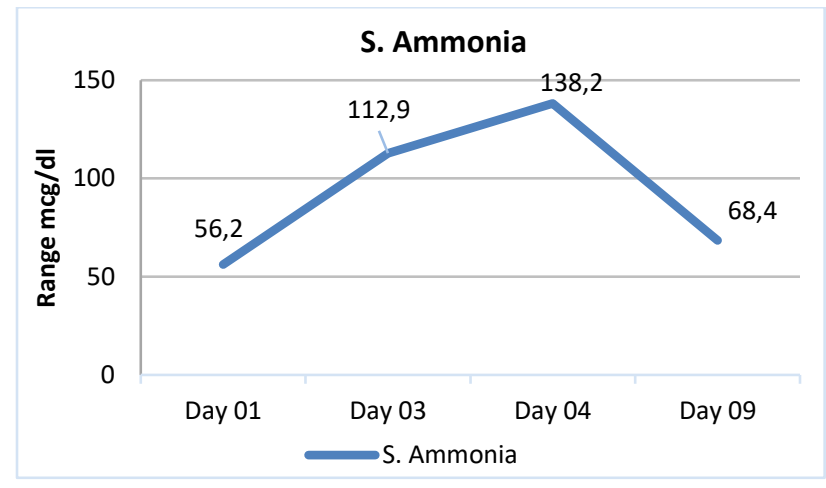

Fig. 1. Elevations in S. Ammonia after Remdesivir therapy and fall after completion of therapy.

On admission the patient was conscious and oriented, Temperature was $99.6 \mathrm{~F}, \mathrm{BP}-150 / 80 \mathrm{mmHg}$, RR-30/min, pulse-80/min and SPO2-98\% on non-rebreather mask 06 $\mathrm{L} / \mathrm{min}$ Oxygen flow. The patient tested positive for COVID19 by Rapid antigen testing and RTPCR.

Her investigations on the day of admission were as follows: $\mathrm{Hb}: 12.2 \mathrm{~g} / \mathrm{dl}$, WBC: 6550 cells/cumm, platelets: 1,50,000 cells/cumm, Creatinine: 1.48 mg/dL, Urea: 43.7 mg/dl, SGPT: 36 U/L, SGOT: 76 U/L, ALP: 31 U/L, Total Bilirubin :0.22 mg/dl, A/G Ration: $2.57 \%$ and inflammatory markers like D-dimer: $4.47 \mu \mathrm{g} / \mathrm{ml}(<0.5)$, ESR: $02 \mathrm{~mm} / \mathbf{h r}$, IL-6: 46.1 Pg/ml and CRP: 65.49 mg/L $(<5.0)$.

She was treated with Injection Remdesivir $200 \mathrm{mg}$ IV on day of admission then given maintenance dose of $100 \mathrm{mg}$ IV for 5 days. Other treatment included Injection Piperacillin $2.25 \mathrm{gm}$ IV thrice a day, Injection Metronidazole $100 \mathrm{CC}$ IV thrice a day, Injection Ceftriaxone $2 \mathrm{gm} \mathrm{IV} \mathrm{once} \mathrm{a} \mathrm{day,}$ Injection Pantoprazole $40 \mathrm{mg}$ IV once a day, Injection Metoclopramide 1 Ampule IV twice a day, Injection LMWH $0.6 \mathrm{CC}$ SC twice a day, Injection Methylprednisolone $80 \mathrm{mg}$ IV once a day, Tablet Azithromycin $500 \mathrm{mg}$ Once a day, Tablet Rifaximin $550 \mathrm{mg}$ twice a day, Tablet Vitamin-C 500 mg twice a day and given other supportive treatment.

On day 3, Laboratory Investigation showed Patient's serum ammonia level was raised to $112.9 \mathrm{mcg} / \mathrm{dl}$ at the time of $3^{\text {rd }}$ dose of Injection Remdesivir. On day 4 again serum ammonia level was raised to $138.2 \mathrm{mcg} / \mathrm{dl}$ at the time of $4^{\text {th }}$ dose of Injection Remdesivir. All possible causes were ruled out. Treatment was completed on day 5, Remdesivir was suspected for Hyperammonemia. On day $9^{\text {th }}$ Repeated serum ammonia level which was lesser then the base line. Despite Remdesivir and steroid therapy, this patient scummed to COVID-19 pneumonia on day $13^{\text {th }}$ of admission.

\section{B. Case II}

A 64 years old hypertensive and diabetic female patient was brought to Emergency Medicine Department of tertiary care hospital with complain of breathing difficulty for 5 days, dry cough for 4 days, fever for 3 days, and loss of appetite for 2 days. No past history of Tuberculosis, COPD, Asthma, IHD, CKD and no surgical past history. Patient was diagnosed with Covid 19 Pneumonitis.

On admission the patient was conscious and oriented, Temperature was $98.6 \mathrm{~F}, \mathrm{BP}-130 / 70 \mathrm{mmHg}$, RR-28/min, pulse-98/min and SPO2-94\% on non-rebreather mask 15 L/min Oxygen flow. The patient tested positive for COVID19 by Rapid antigen testing and RTPCR.
Her investigations on the day of admission were as follows: $\mathrm{Hb}$ : $13 \mathrm{~g} / \mathrm{dl}$, WBC: 8300 cells/cumm, platelets: 3,14,000 cells/cumm, Creatinine: $0.70 \mathrm{mg} / \mathrm{dL}$, Urea:52.3 mg/dl, SGPT: 16 U/L, SGOT: 29 U/L, ALP: 100 U/L, Total Bilirubin :0.70 mg/dl, A/G Ration: $1.38 \%$ and inflammatory markers like D-dimer:2.08 $\boldsymbol{\mu g} / \mathbf{m l}(<0.5)$, ESR: 120 mm/hr., IL-6: 197.9 Pg/ml and CRP:348.83 mg/L (<5.0).

She was treated with Injection Remdesivir $200 \mathrm{mg}$ IV on day of admission then given maintenance dose of $100 \mathrm{mg}$ IV for 5 days. Other treatments included, Injection Meropenem $2 \mathrm{gm}$ IV thrice a day, Injection Levofloxacin $750 \mathrm{mg}$ IV once a day, Injection Pantoprazole $40 \mathrm{mg}$ IV twice a day, Injection Ondansetron $4 \mathrm{mg}$ IV thrice a day, Injection Heparin 25000 IU IV Infusion, Injection Lantus 14 Units SC twice a day, Tablet Aspirin 75 Once a day, Tablet Metoprolol $25 \mathrm{mg}$ twice a day, Tablet Vitamin-C $500 \mathrm{mg}$ thrice a day, Injection Tocilizumab $400 \mathrm{mg}$ IV was given after $1^{\text {st }}$ day of admission, Injection Dexamethasone $8 \mathrm{mg}$ IV thrice a day was given after $4^{\text {th }}$ day of admission.

On day 4 patient developed mild Neurological symptoms. On day 5, Laboratory Investigation showed Patient's serum ammonia level was raised. Treatment was completed on day 5. Drug Injection Remdesivir was suspected for Hyperammonemia.

On day $13^{\text {th }}$ Repeated serum ammonia level which was lesser then the base line. Patient was discharged with stable condition on day $15^{\text {th }}$.

\section{DISCUSSION}

Remdesivir is an anti-viral agent that has shown a significant inhibitory effect in vivo and in vitro studies against SARS-CoV-2 and appears to be ahead of other repurposed drugs being tried for the treatment of COVID-19 [5]. FDA and European Medicines Agency (EMA) has currently authorized Remdesivir only in severe COVID-19 infection in both adults and children [5]. The safety profile of Remdesivir in COVID-19 is incompletely characterized in COVID-19 While the safety data from the previous use during acute Ebola Virus Disease (EVD) suggest no specific alarm, COVID-19 differs profoundly in its clinical characteristics from EVD. Nevertheless, hitherto no safety findings allow Remdesivir to be used in COVID-19, under a proper pharmacovigilance [5]. Special attention should be given for disproportionate rise in ALT or decrease in GFR, during the treatment with Remdesivir [5].

Remdesivir has an elimination half-life of 1 hour following a single 30-minute intravenous infusion. Under the same conditions, the elimination half-lives of the Remdesivir metabolites GS-441524 and GS-704277 are 27 hours and 1.3 hours, respectively [6]. The nucleoside triphosphate metabolite has a half-life of $14 \mathrm{~h}$ in non-human primates. The nucleoside triphosphate metabolite has a half-life of approximately 20 hours in humans [6].

These cases have raised concerns regarding SRIH in patients with COVID-19. Hyperammonemia is lifethreatening condition which can affect patients at any age [7]. Elevations of ammonia in plasma indicate its increased production and/or decreased detoxification [7]. The hepatic urea cycle is the main pathway to detoxify ammonia; it can 
be defective due to an inherited enzyme deficiency or secondary to accumulated toxic metabolites or substrate depletion [7]. A variety of environmental causes and medications may also lead to ammonia toxicity [8].

In these two case reports, COVID-19 patients developed hyperammonemia during Remdesivir treatment. Laboratory finding suggested that sudden rise in serum ammonia level on $5^{\text {th }}$ day in patient $1,4^{\text {th }}$ day in patient 2 during course of admission which returned to base line after treatment was completed. Causal relationship between drug and event suggested that it was possibly due to the administration of Remdesivir drug.

Overall, there is a need to continually monitor ADR arising from Remdesivir to provide future guidance. There is a role for Drug and Therapeutic Committees (DTCs) in hospitals to enhance ADR reporting as well as continue to promote evidence-based medicine (EBM) to optimize treatment for patients with COVID-19 and other diseases [9]. Solidarity Trial of WHO found that treatment of Remdesivir had little or no effect on overall mortality, initiation of ventilation and duration of hospital stay in hospitalized patients [10]. This demand updating physicians on the effectiveness and safety of Remdesivir as new information becomes available in line with activities and recommendations for managing the entry of new medicines into clinical care [9]. physicians should be aware of this possible association and perform serum ammonia level monitoring when prescribing Remdesivir.

\section{CONCLUSION}

The use of the novel antiviral Remdesivir in the treatment of COVID-19 pneumonia may put patients at risk of SRIH. In presented two cases, the Hyperammonemia resolved by the time therapy was completed. More data on the safety of Remdesivir is needed especially when our country is anticipating a third wave of the pandemic.

\section{REFERENCES}

[1] Lo M.K., Jordan R., Arvey A. GS-5734 and its parent nucleoside analog inhibit filo-, pneumo-, and paramyxoviruses. Sci Rep. 2017; 7:43395.

[2] Sheahan T.P., Sims A.C., Graham R.L. Broad-spectrum antiviral GS5734 inhibits both epidemic and zoonotic coronaviruses. Sci Transl Med. 2017;9.

[3] Agostini M.L., Andres E.L., Sims A.C., Graham R.L., Sheahan T.P., Lu X. Coronavirus susceptibility to the antiviral remdesivir (GS-5734) is mediated by the viral polymerase and the proofreading exoribonuclease. mBio. 2018;9/

[4] Summary on compassionate use remdesivir Gilead. https://www.ema.europa.eu/en/documents/other/summarycompassionate-use-remdesivir-gilead_en.pdf (Accessed May 5, 2020)/

[5] Singh AK, Singh A, Singh R, Misra A. Remdesivir in COVID-19: A critical review of pharmacology, pre-clinical and clinical studies. Diabetes Metab Syndr. 2020;14(4):641-648.

[6] Bethesda (MD): National Library of Medicine (US), National Center for Biotechnology Information; 2004-. PubChem Compound Summary for CID 121304016, Remdesivir; [cited 2021 June 6]. Available from: https://pubchem.ncbi.nlm.nih.gov/compound/Remdesivir/

[7] Häberle, J(2011). Clinical practice: the management of hyperammonemia. European Journal of Pediatrics, 170(1):21-34.

[8] Auron A, Brophy PD. Hyperammonemia in review: pathophysiology, diagnosis, and treatment. Pediatr Nephrol. 2012 Feb;27(2):207-22.

[9] Charan J, Kaur RJ, Bhardwaj P, et al. Rapid review of suspected adverse drug events due to remdesivir in the WHO database; findings and implications. Expert Rev Clin Pharmacol. 2021;14(1):95-103.

[10] Pan H, Peto R, Henao-Restrepo AM, Preziosi MP, Sathiyamoorthy V, Abdool Karim Q, Alejandria MM, Hernández García C, Kieny MP, Malekzadeh R, Murthy S, Reddy KS, Roses Periago M, Abi Hanna P, Ader F, Al-Bader AM, Alhasawi A, Allum E, Alotaibi A, AlvarezMoreno CA, Appadoo S, Asiri A, Aukrust P, Barratt-Due A, Bellani S, Branca M, Cappel-Porter HBC, Cerrato N, Chow TS, Como N, Eustace J, García PJ, Godbole S, Gotuzzo E, Griskevicius L, Hamra R, Hassan M, Hassany M, Hutton D, Irmansyah I, Jancoriene L, Kirwan J, Kumar S, Lennon P, Lopardo G, Lydon P, Magrini N, Maguire T, Manevska S, Manuel O, McGinty S, Medina MT, Mesa Rubio ML, MirandaMontoya MC, Nel J, Nunes EP, Perola M, Portolés A, Rasmin MR, Raza A, Rees H, Reges PPS, Rogers CA, Salami K, Salvadori MI, Sinani N, Sterne JAC, Stevanovikj M, Tacconelli E, Tikkinen KAO, Trelle S, Zaid H, Røttingen JA, Swaminathan S. Repurposed Antiviral Drugs for Covid-19 - Interim WHO Solidarity Trial Results. N Engl J Med. 2021 Feb 11;384(6):497-511.

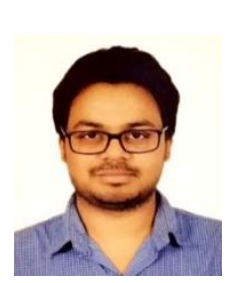

Sagar R. Bhimani was born in Gujarat, India in 1993.He had done his Bachelor of Medicine and Bachelor of Surgery (M.B.B.S) degree from Gujarat Adani institute of Medical Sciences, Gujarat, India in 2017. He is now Pursuing his M.D in Pharmacology at Smt. NHL Municipal Medical College, Ahmedabad, India. 